\title{
Biochemical and Histologic Pathology in an Infant with Cross-Reacting Material (Negative) Pyruvate Carboxylase Deficiency
}

\author{
LAWRENCE T. K. WONG, A. GEORGE F. DAVIDSON, DEREK A. APPLEGARTH, \\ JAMES E. DIMMICK, MARGARET G. NORMAN, JENNIFER R. TOONE, GORDON PIRIE, AND \\ JONATHAN WONG \\ Department of Paediatrics and Pathology, British Columbia Children's Hospital, University of British Columbia, \\ Vancouver, British Columbia, Canada
}

\begin{abstract}
An infant with the acute neonatal form of pyruvate carboxylase deficiency (cross-reacting material negative) presented with severe intractable lactic acidosis within $4 \mathrm{~h}$ after birth. He also had hyperammonemia, hypercitrullinemia, and hyperlysinemia. Plasma glutamine was not elevated. He had a rapidly deteriorating clinical course with severe liver dysfunction, repeated septicemia and seizures; he was comatose and was on a ventilator throughout; death occurred at 8 wk of age. Skin fibroblast study confirmed the enzyme deficiency. Detailed biochemical parameters and histopathology of the brain and liver are presented. The evidence from this infant suggests that disturbances of intracellular oxaloacetate levels as a result of the primary enzyme defect might also contribute to deficiency in ATP generation which may explain the various other biochemical changes and liver pathology. ( $P e-$ diatr Res 20: 274-279, 1986)
\end{abstract}

\section{Abbreviations}

PC, pyruvate carboxylase

CRM, cross-reacting material

$\alpha \mathbf{K G}, \alpha$-ketoglutarate

Patients with PC deficiency have presented with lactic acidosis. There are two main forms of the disease-the acute neonatal and infantile forms. The acute neonatal form of this condition was first described by Coude and coworkers $(1,2)$. Their patients followed a rapidly malignant course and died within the first months of life. Besides severe intractable lactic acidosis (levels of 10 to $20 \mathrm{mM}$ ), there were other biochemical abnormalities including hyperammonemia (in the range of 100 to $300 \mu \mathrm{M}$ ), citrullinemia (up to $400 \mu \mathrm{M}$ ), lysinemia (up to $800 \mu \mathrm{M}$ ), elevated lactate-pyruvate ratio (range 50-100), and a lowered $\beta$-hydroxy butyrate-acetoacetate ratio (less than 1). Skin fibroblasts cultured from patients with this acute neonatal form of PC deficiency have recently been shown to have complete absence of a protein recognized by anti-PC antibodies, i.e. they were CRM negative (3). Patients presenting with milder infantile forms of the disease had immunologically detectable PC (i.e. CRM positive). The two groups were indistinguishable by assays of enzyme activity.

We describe the detailed clinical and biochemical course together with the histopathology of one PC-deficient (CRM nega-

Received August 19, 1985; accepted November 13, 1985.

Correspondence and reprint requests to Dr. L. T. K. Wong, Biochemical Diseases Clinical Service, Children's Hospital, 4480 Oak Street, Vancouver, BC, Canada V6H 3V4 tive) patient. This patient is one of the group described earlier (3). Because only an extremely abbreviated clinical description was possible in the earlier paper and because we believe that the symptomatology as well as the biochemical aberrations and histopathology have important implications we therefore present this patient's findings in more detail.

\section{METHODS}

Blood ammonia, lactate, and bilirubin were assayed by the Dupont aca (13); electrolytes by Beckman ASTRA system; blood $\mathrm{pH}$ and bicarbonate by the Corning $\mathrm{pH} /$ Blood Gas Analyser (13); and serum alkaline phosphatase, aspartate transaminase, alanine transaminase, by the Technicon RA-1000 (13). Plasma and urinary amino acids were assayed by methods previously described $(4,5)$. Urine organic acids were examined using the Hewlett-Packard 5992A GC/MS (6). Plasma acetoacetate and $\beta$ hydroxybutyrate were assayed according to the micromethod of Persson (7). Plasma pyruvate was assayed using Sigma kit no. 726 (these are trademarks of the respective companies, protected by appropriate patent and copyright legislation).

\section{CASE REPORT}

A male infant was transferred to the Intensive Care Unit at BC Children's Hospital at 1 day of age because of severe metabolic acidosis. He was the second child of a healthy unrelated young couple. A 3-yr-old brother was in good health. The infant was born at 38 wk gestation by elective caesarean section. He was well at birth, with a 1-min Apgar score of 8 . Birth weight was $3.3 \mathrm{~kg}$, crown-heel length $55 \mathrm{~cm}$, and head circumference 36 $\mathrm{cm}$. Four hours postpartum he developed tachypnea which rapidly worsened with grunting respirations and pallor. Profound metabolic acidosis was noted with blood pH 7.22, $\mathrm{pCO}_{2} 25 \mathrm{~mm}$ $\mathrm{Hg}$, and bicarbonate $12 \mathrm{mEq} /$ liter. Despite repeated intravenous sodium bicarbonate he remained severely acidotic and at $7 \mathrm{~h}$ postpartum his blood $\mathrm{pH}$ had fallen to $7.04, \mathrm{pCO}_{2} 37 \mathrm{~mm} \mathrm{Hg}$ and bicarbonate was $10 \mathrm{mEq} /$ liter. He was intubated, ventilated, and transferred to this hospital.

On examination the infant appeared to have a small face with a large cranial vault. He had gasping respirations with marked intercostal and sternal indrawing. Bilateral inspiratory fine crepitations were audible in the chest. The abdomen was scaphoid with liver enlarged and palpable $3 \mathrm{~cm}$ below the costal margin.

Laboratory results on admission showed significant increases in plasma lactate, ammonia, citrulline, alanine, lysine, proline, and tyrosine. He had normal values of plasma glutamine, ornithine, arginine, methionine, and glutamic and aspartic acids were low (Table 1). Urinary organic acids showed large quantities of lactate, $\beta$-hydroxybutyrate, and $\alpha$-hydroxyisobutyrate. 
Over the next $72 \mathrm{~h}$, correction of the severe metabolic acidosis required continuous sodium bicarbonate infusion of over $3 \mathrm{mEq} /$ $\mathrm{h}$ plus assisted ventilatory support.

During the next $2 \mathrm{wk}$ the infant continued to need ventilatory support. Maintenance of his blood $\mathrm{pH}$ in the normal range required bicarbonate infusion averaging $1.2 \mathrm{mEq} / \mathrm{h}$. Despite this, other biochemical abnormalities persisted (Figs. 1 and 2). Blood ammonia remained high (100 to $300 \mu \mathrm{M}$ ); lactate was 10 to 20 $\mathrm{mM}$; glutamine remained low and fell to below $50 \mu \mathrm{M}$. Citrulline levels gradually decreased but were still above the normal range until just before death. Proline and lysine continued to be elevated while glutamic and aspartic acids remained low. There was also decrease of alanine, tyrosine, and arginine (Table 1).

On day 18 nasogastric feeding was instituted using a low protein formula containing $20 \%$ Caloreen and Similac 68 , to supply $120 \mathrm{kcal}, 0.5 \mathrm{~g}$ protein, and $80 \mathrm{ml}$ fluid per $\mathrm{kg}$ per day. This had no significant effect on the lactic acidosis (Fig. 1) but

Table 1. Initial levels of plasma lactate $(m M)$, ammonia $(\mu M)$, and amino acids $(\mu M)$

\begin{tabular}{lccccc}
\hline & Day 2 & Day 12 & Day 21 & Day 45 & $\begin{array}{c}\text { Newborn } \\
\text { normal range }\end{array}$ \\
\hline Lactate & 20.7 & 14.8 & 16.3 & 12.4 & $0.5-2.2$ \\
Ammonia & 136 & 296 & 409 & 15.4 & up to 40 \\
Proline & 689 & 195 & 375 & 111 & $144-329$ \\
Alanine & 733 & 52 & 93 & 183 & $132-329$ \\
Citrulline & 390 & 390 & 112 & 13 & $3-36$ \\
Tyrosine & 288 & 124 & 121 & 127 & $32-124$ \\
Lysine & 664 & 270 & 375 & 159 & $71-272$ \\
Glutamic acid & 10 & Trace & 8 & 18 & $30-103$ \\
Glutamine & 462 & 260 & 85 & 60 & $243-822$ \\
Methionine & 46 & 28 & 17 & 9 & $6-36$ \\
Arginine & 84 & Trace & 26 & Trace & $17-119$ \\
Ornithine & 45 & 14 & 22 & 7 & $38-207$ \\
Aspartic acid & 7 & 6 & 7 & 0 & $0-8$
\end{tabular}

PATIENT A.Y.
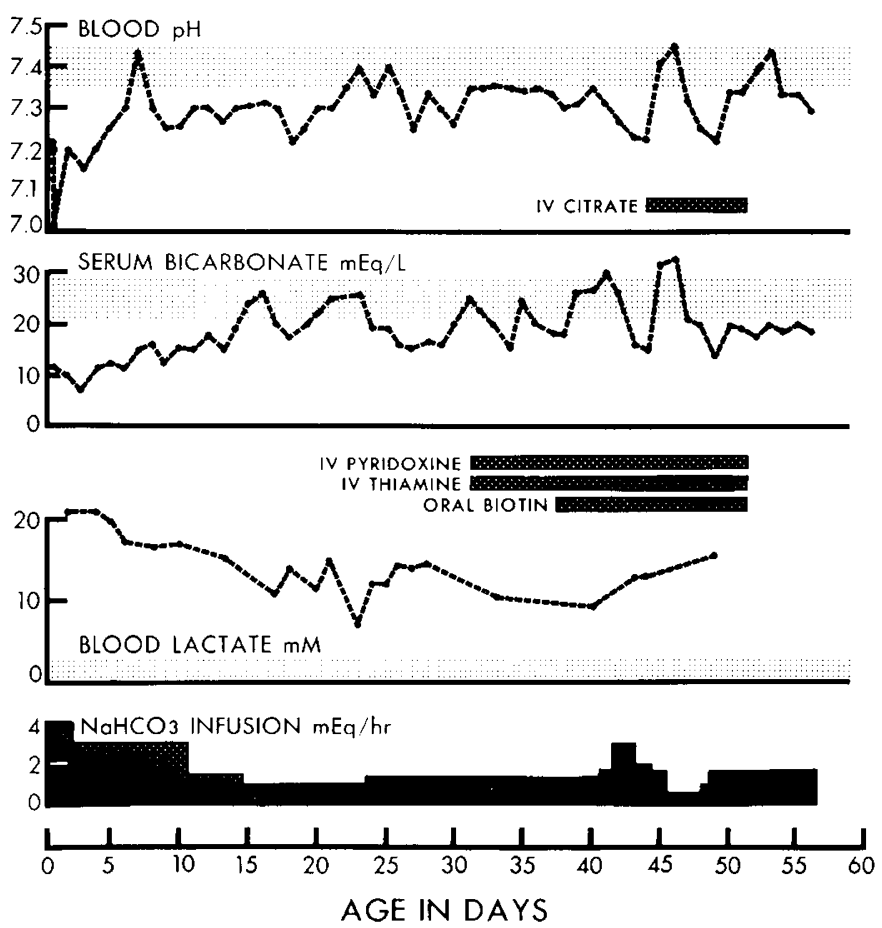

Fig. 1. Blood pH, serum bicarbonate, and blood lactate levels in infant. Dotted areas indicate normal range for age. Therapy with sodium bicarbonate $\left(\mathrm{NaHCO}_{3}\right)$, pyridoxine, thiamine, biotin, and citrate is denoted by the hatched areas.
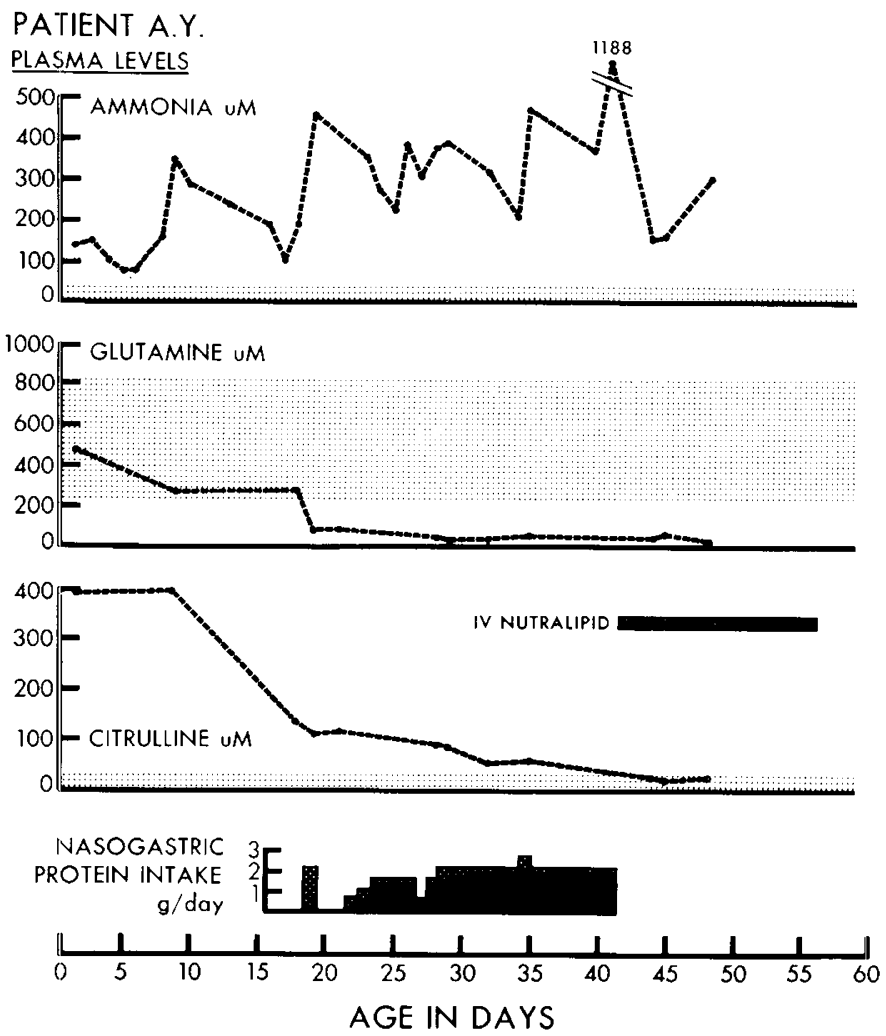

Fig. 2. Plasma ammonia, glutamine, and citrulline in the infant. Dotted areas indicate normal range for age. Nutritional support with nasogastric feeding (see text) and intravenous Nutralipid are shown.

resulted in a significant increase of ammonia, to $460 \mu \mathrm{M} 2$ days afterward. Further decreases of glutamine and citrulline occurred despite the feeding.

A therapeutic trial of pyridoxine ( $200 \mathrm{mg}$ daily), thiamine (200 $\mathrm{mg}$ daily), and biotin ( $15 \mathrm{mg}$ daily) did not result in any significant clinical or biochemical responses (Fig. 1). On day 32 plasma lactate was $10.2 \mathrm{mM}$ and pyruvate $0.068 \mathrm{mM}$ while acetoacetate was $340 \mu \mathrm{M}$ and D- $\beta$-hydroxybutyrate was $65 \mu \mathrm{M}$. Significant exacerbation of the acidosis was noted on day 42 and required an increase in the infusion of sodium bicarbonate to more than $4 \mathrm{mEq} / \mathrm{h}$. At this time intravenous sodium citrate was instituted. Nine $\mathrm{ml}$ of a $4 \%$ solution (i.e. $1.4 \mathrm{mEq}$ ) was given every $6 \mathrm{~h}$. This resulted in dramatic correction of the acidosis and it was possible to reduce the sodium bicarbonate infusion to $0.4 \mathrm{mEq} /$ h (Fig. 1).

The clinical course was aggravated by the following complications. Repeated episodes of septicemia occurred due to (on separate occasions) gram-positive cocci, (Day 27), Escherichia coli (day 33) and staphylococcus epidermidis (day 49). Repeated courses of intravenous antibiotics were given. Chronic unrelenting coagulopathy with prolonged prothrombin time and partial thromboplastin time (both were more than $120 \mathrm{~s}$ ), and hypofibrinogenemia $(14 \mathrm{mg} / 100 \mathrm{ml})$ were present. There were no fibrin degradation products, and factor VIII concentration was normal but there was a slight reduction of factor VII. Repeated transfusion of fresh frozen plasma and cryoprecipitate given almost daily failed to correct the coagulopathy, nor did vitamin $\mathrm{K}$. Despite the coagulopathy bleeding was not a problem. Hepatic dysfunction was evident; the infant was jaundiced on admission with a total bilirubin of $7.2 \mathrm{mg} / \mathrm{dl}$ and direct of $0.6 \mathrm{mg} / \mathrm{dl}$. Jaundice increased with a total bilirubin of $14.9 \mathrm{mg} / \mathrm{dl}$ and direct bilirubin of $5.5 \mathrm{mg} / \mathrm{dl}$ just prior to the infant's death. On admission serum lactate dehydrogenase and aspartate transaminase were twice the normal values whereas alanine transaminase was within normal limits. Central nervous system dysfunction was also evident. The infant showed very little spontaneous activity 
and had minimal response to stimulation. A large head was noted on admission and head sonogram showed mild to moderate dilatation of ventricles with cystic areas in the subependymal region particularly on the left. This finding was confirmed by CT scan which revealed porencephalic cysts with periventricular leukomalacia and ventricular dilatations. These changes were suggestive of an intrauterine subependymal bleed with intraventricular extension. Transient seizure activity was noted on day 14. EEG showed left temporal and generalized seizure activities. There was slow background activity. These EEG findings persisted even when he was maintained on Phenobarbital $10 \mathrm{mg}$ per $\mathrm{kg}$ body weight. Bronchopulmonary dysplasia as indicated by typical $\mathrm{x}$-ray occurred after $3 \mathrm{wk}$ of continuous ventilatory support. There was marked hyperinflation of the left lung and patches of atelectasis and infiltrate were noted in both lung fields.

Hypernatremia was a constant problem, perhaps because of the constant need for intravenous sodium bicarbonate. Serum sodium was intermittantly as high as $161 \mathrm{mg} / \mathrm{liter}$. The infant's general condition deteriorated. He developed septicemia and worsening pulmonary status and died at 8 wk of age.

Skin fibroblast $P C$ studies. These studies have been previously reported (3) (case 8). The patient was shown to be CRM negative for PC. A later prenatal diagnosis in this family has also been described (11).

\section{HISTOPATHOLOGY}

A surgical biopsy of liver obtained at 3 wk of age was fixed immediately for light microscopy and electron microscopy, and snap frozen in liquid nitrogen for biochemistry and histochemistry.

Microscopic examination of the liver revealed cytoplasmic and canalicular cholestasis, mild ductular proliferation with portal septal fibrosis, and pseudoglandular rearrangement of hepatocytes (Figs. 3 and 4). Hepatocytes were moderately swollen, many had prominent nucleoli and nuclei, a few were in mitosis and none was necrotic. Lipid droplets, predominantly large and in moderate amount, were present in the mid-zone and perivenular regions. Succinate dehydrogenase activity was normal histochemically; iron stores slightly increased in Kupffer cells, and glycogen was present but not prominent in hepatocytes. On electron microscopy the major ultrastructural change not evident by light microscopy involved mitochondria (Fig. 5). These or- ganelles were normal in number, but were slightly pleomorphic and had increased matrix density, increased matrix granule size, dilatation of the intracristal space, and slight widening of the space between the mitochondrial outer membranes. Mitochondrial alterations were present uniformly in the lobule and through the electron microscopic grid sections. Peroxisomes were present and appeared normal.

Autopsy examination revealed that the liver was firm, smooth, green, and twice the normal weight. Microscopic pathology was similar to the surgical biopsy specimen but the cholestasis, steatosis, septal fibrosis, and nucleolar and nuclear enlargement were more pronounced. Hepatocellular necrosis and inflammation were again absent.

The brain weight at autopsy was $475 \mathrm{~g}$ (normal for crown-heel $360 \mathrm{~g}$ ). Bilaterally symmetrical cavitated infarcts 0.5 to $1 \mathrm{~cm}$ in diameter were present in the frontal lobes and extended from the frontal tip to the level of the genu of the corpus callosum. The parenchyma between the cortex and the central nuclei and ventricles was gray and sclerotic on gross examination and showed diffuse gliosis microscopically. Old cavitated hemorrhages $0.5 \mathrm{~cm}$ in diameter were present in the germinal eminences over the caudate nuclei bilaterally. There was patchy ependymal loss and hemosiderosis of the ventricular system secondary to rupture of the hemorrhages into the ventricles. In addition synechiae had formed in the left lateral ventricle. Extension of the hemorrhage into the subarachnoid space at the base of the brain had resulted in a chronic arachnoiditis which had obstructed cerebrospinal fluid flow and resulted in hydrocephalus with enlargement of the lateral ventricles, thinning of the corpus callosum, and attenuation of the septum pellucidum. An old cavitated hemorrhage $0.5 \mathrm{~cm}$ in diameter was present in the right cerebellar hemisphere. No myelin was present in the hemispheres. There was myelin pallor and spongy change in the posterior columns of the spinal cord, medial leminiscus, and brachium conjunctivium. The liver failure had resulted in the presence of Alzheimer type II astrocytes in the gray matter.

Other relevant pathology from autopsy examination included nonacanthotic hyperkeratosis of skin, bronchopulmonary dysplasia of lungs, and advanced thymic involution.

\section{DISCUSSION}

This infant with confirmed CRM negative PC deficiency, followed a malignant clinical course similar to that of infants

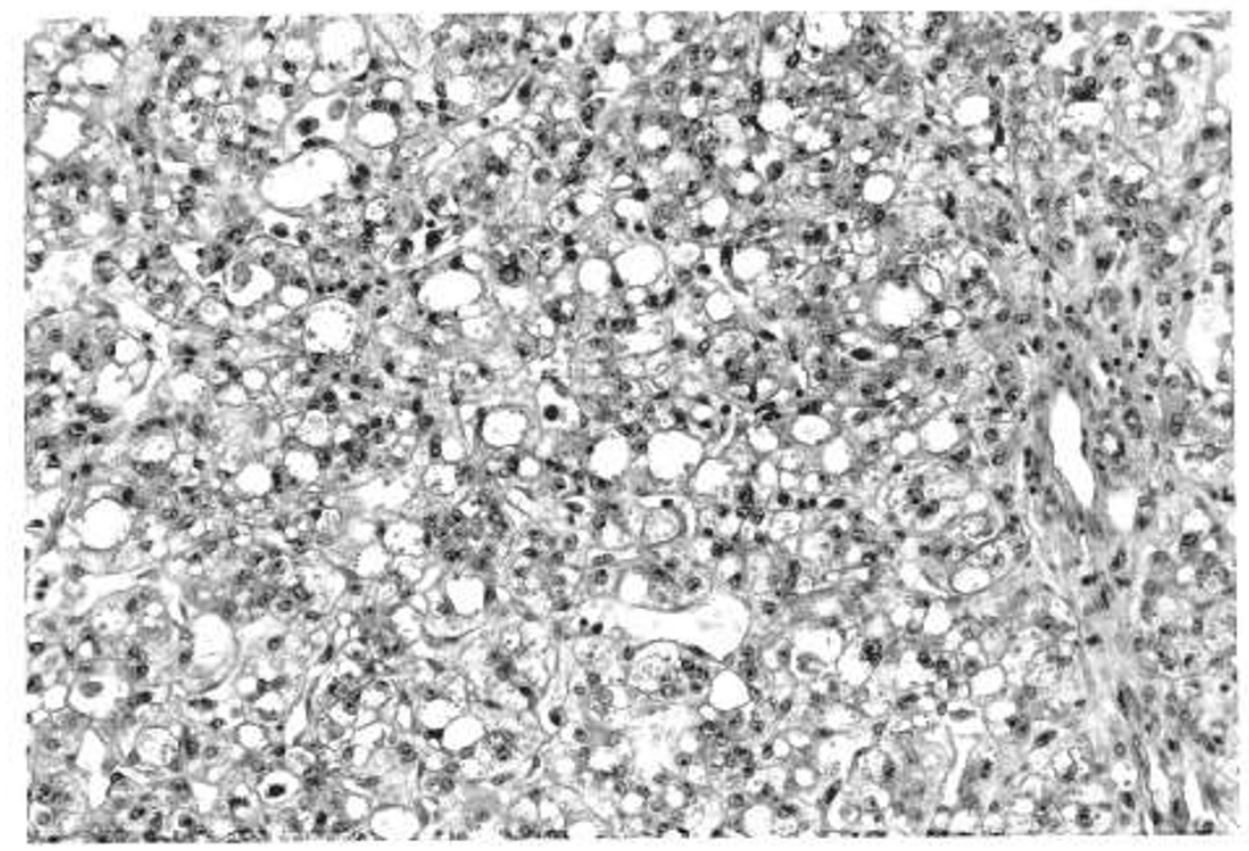

Fig. 3. Liver hematoxylin and eosin; original magnification $\times 100$. Hepatocytes are swollen, cholestasis and steatosis are present. 


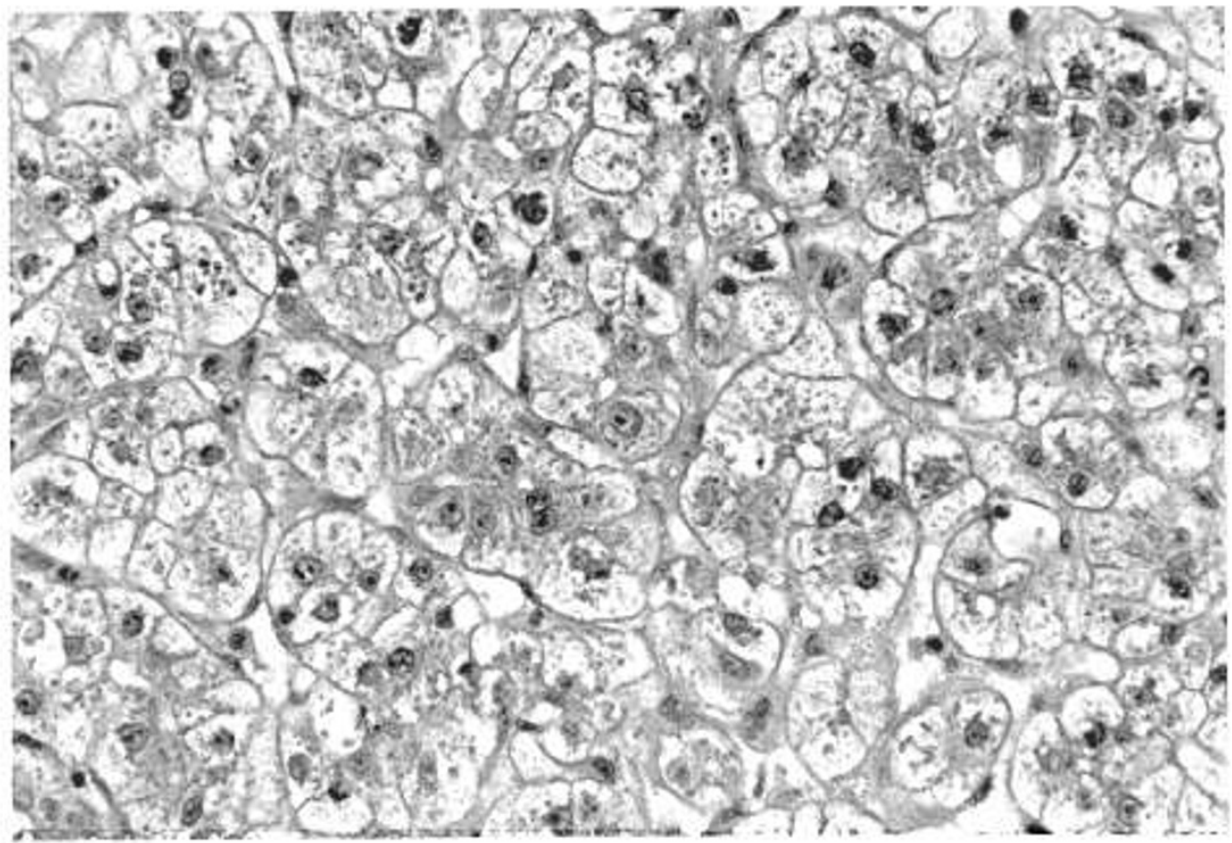

Fig. 4. Liver hematoxylin and eosin; original magnification $\times 400$. Note pseudoglandular arrangements of hepatocytes and the prominence of nucleoli.

reported from France $(1,2)$. The child died at 8 wk of age. He had unrelenting lactic acidosis associated with a high plasma lactate/pyruvate ratio of approximately 150 (normal 10-25). The ratio of plasma acetoacetate to $\beta$-hydroxybutyrate (done on one occasion only) was also high. The value was 5.2 (normal $0.4-$ 2.0). After the initial repeated intravenous pushes of sodium bicarbonate, blood $\mathrm{pH}$ was maintained at around 7.30 with a bicarbonate infusion rate of $3 \mathrm{mEq} / \mathrm{h}$ during the first 10 days. Subsequently for a period of $4 \mathrm{wk} 1 \mathrm{mEq} / \mathrm{h}$ was required. There was no response to high doses of pyridoxine, thiamine, and biotin. With later progressive clinical deterioration bicarbonate requirement again increased to $3 \mathrm{mEq} / \mathrm{h}$. At this stage the acute beneficial effect of sodium citrate was tried. Citrate infusion did indeed spare the need for bicarbonate but, it was difficult to assess its effects on other metabolic parameters, since it was at this time that continuous nasogastric feeding was stopped and intravenous lipid emulsion begun. The role of citrate, arginine, and aspartate (or oxaloacetate) in the therapy of this disease needs to be evaluated earlier in the course of the disease in future cases.

As far as the biochemistry of this child is concerned, several interesting phenomena were observed. One striking initial observation was that there was an increased amount of citrulline in the plasma and this, in conjunction with the hyperammonemia, led to consideration of a diagnosis of primary citrullinemia. However, the plasma glutamine concentration, which normally parallels an increase in blood ammonia, was below normal. Plasma alanine level was initially high but quickly became normal or below normal even when plasma lactate was at least 6or 7-fold increased. There was a normal level of pyruvate despite the increased lactate and there was a decreased ratio of $\beta$ hydroxybutyrate to acetoacetate (in contrast to the lactate to pyruvate ratio where the lactate was proportionately increased more than the pyruvate). In the case of $\beta$-hydroxybutyrate and acetoacetate it was the oxidized form (acetoacetate) which was increased over the reduced form ( $\beta$-hydroxybutyrate) whereas the oxidized form (pyruvate) was lower than the reduced form (lactate).

We believe that the above noted unusual biochemistry can be explained as a consequence of the complete absence of PC activity. This child had no immunologically detectable PC suggesting complete absence of the enzyme protein. Therefore no oxaloacetate could be produced from pyruvate. The lack of oxaloacetate has important consequences as this would directly affect other reactions (Fig. 6). Apart from the obvious increase of lactate concentration, the metabolic pathways most likely to be affected are the Kreb's (tri-carboxylic) citric acid cycle which is needed for ATP generation, the disposal of ammonia through the generation of glutamate/glutamine and the urea cycle, and the dicarboxylate transport system which helps to regulate the NADH/NAD ratio.

Within the mitochondria increased production of acetyl CoA would occur as the result of accumulation of pyruvate. However, the low level of oxaloacetate would not allow rapid utilization of acetyl CoA by the citric acid cycle. This would affect the generation of ATP and thus would affect those energy-dependant reactions requiring ATP. Also a larger amount of acetoacetate might be expected to be formed from acetyl CoA. One would then expect to see metabolic attempts to restore or conserve the oxaloacetate concentrations. Aspartate is transformed to oxaloacetate using aspartate aminotransferase and in doing so $\alpha \mathrm{KG}$ which is already at a low level due to the lack of oxaloacetate, would be further depleted. The combined effect of low $\alpha \mathrm{KG}$ and aspartate would be to compromise ammonia disposal. With less $\alpha \mathrm{KG}$ available for the various transamination reactions less glutamate would be formed and consequently less ammonia could be transformed into glutamine (a conventional "ammonia sink"). In this infant glutamine, which initially was normal (possibly as a carry over of maternal levels), decreased rapidly to subnormal level. Since aspartate is also needed for argininosuccinate synthesis in the urea cycle, this reaction may also be compromised leading to high citrulline values as was seen persistently in this infant. Furthermore, the above two reactions in the formation of glutamine and argininosuccinate together with the first step in the urea cycle (carbamyl phosphate synthesis) are all ATP-dependant reactions. These would probably be further compromised as a result of the deficient ATP generation by the citric acid cycle due to the restricted amount of oxaloacetatean obligatory component of that cycle. All these therefore would act together to explain the unrelenting hyperammonemia in this baby. The progressive later drop in plasma citrulline concentrations could be partly explained on the basis of decreased carbamyl phosphate synthetase activity due to ATP depletion, although other factors such as decreased plasma arginine and protein restriction likely also contribute to this.

Another aspect of oxaloacetate metabolism is its involvement 


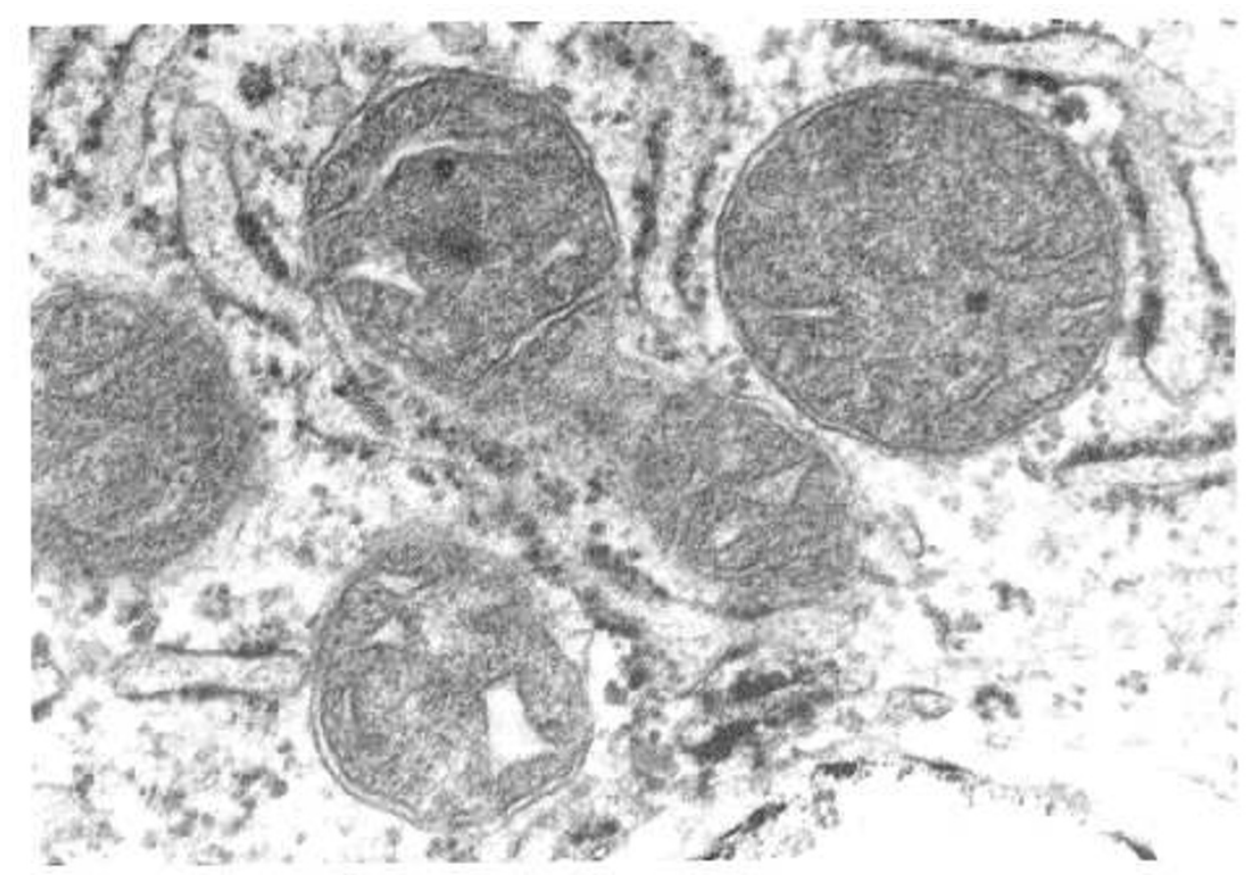

Fig. 5. Liver mitochondria: magnification $\times 90,000$. Note increased matrix density and dilated intracristal space.

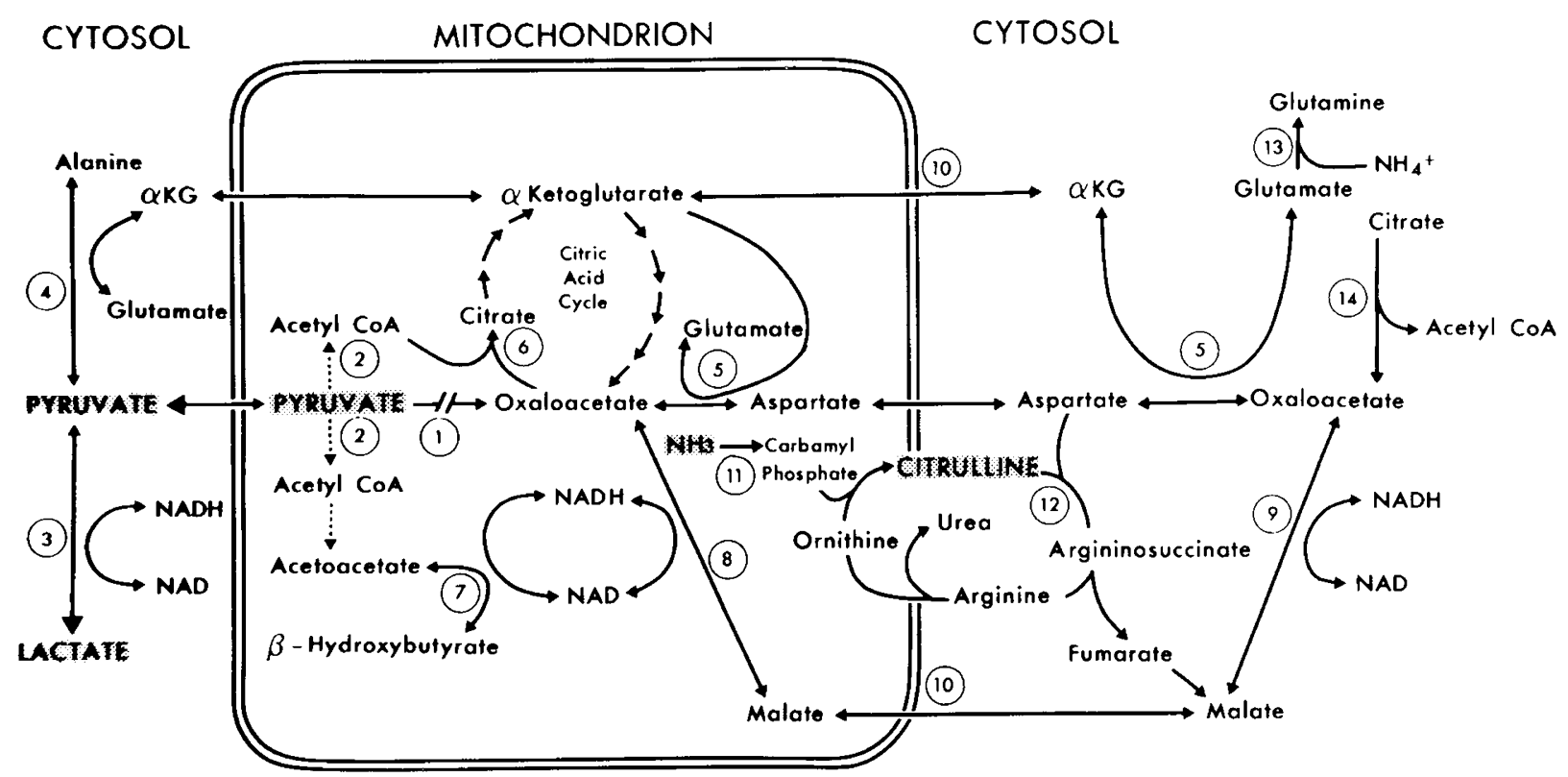

Fig. 6. Biochemical reactions in mitochondrion and cytosol affected by PC deficiency. Significantly elevated compounds are indicated by emphasized print. The enzymes are: (1) PC; (2) pyruvate dehydrogenase complex; (3) lactate dehydrogenase; (4) alanine aminotransferase; (5) aspartate aminotransferase; (6) citrate synthase; (7) $\beta$-hydroxybutyrate dehydrogenase; (8) malate dehydrogenase (mitochondria); (9) malate dehydrogenase (cytosol); (10) dicarboxylate transport system; (11) carbamylphosphate synthetase; (12) argininosuccinic synthetase; (13) glutamine synthetase; (14) citrate cleavage enzyme.

in the regulation of NAD/NADH ratios through its conversion to malate by malate dehydrogenase. The lowered levels of oxaloacetate would lead to lower levels of malate compromising the shuttle of reducing equivalents across the mitochondrial membrane by affecting the dicarboxylate transport system (Fig. 6). This transport system involves transport of malate from the cytosol across the mitochondrial membrane into the mitochondrial matrix. Malate is then converted to oxaloacetate and $\alpha \mathrm{KG}$ which is then transported back into the cytosol. The net effect of this shuttle is to take a reduced compound (malate) into the mitochondrion and bring back into the cytosol an oxidized compound $(\alpha \mathrm{KG})$. This system depends on the oxidation of
$\mathrm{NADH}$ to NAD in the cytosol and reduction of NAD to NADH in the mitochondrion. Since there is presumably not enough oxaloacetate for the malate dehydrogenase reaction to function normally, this would result in an increased cytosol NADH/NAD ratio with the opposite situation inside the mitochondrion. The effect of an increased cytosol NADH/NAD ratio would be to increase the lactate level at the expense of pyruvate (as was observed). In the mitochondrion one would expect a decreased $\mathrm{NADH} / \mathrm{NAD}$ ratio and this could account for the fact that the plasma acetoacetate concentration was much higher than that of $\beta$-hydroxybutyrate because $\beta$-hydroxybutyrate dehydrogenase is a mitochondrial enzyme which requires NADH. A similar ratio 
of acetoacetate/ $\beta$-hydroxybutyrate was reported in the French patients $(1,2)$.

Alanine is normally derived from pyruvate and glutamate through the action of alanine aminotransferase. Therefore it is interesting to observe that the plasma alanine which was initially elevated quickly became subnormal. This probably reflects the combined effect of lowered glutamate and pyruvate (due to preferential conversion to lactate as a result of the increased cytosol NADH/NAD ratio).

Through the action of citrate cleavage enzyme in the cytosol, citrate can contribute to oxaloacetate. This might explain the transient beneficial effect of citrate infusion. Citrate itself can provide the carbon skeleton needed for increased efficiency of the Kreb's citric acid cycle and therefore probably substituted for oxaloacetate in the ATP generation systems. This suggests the possibility of future therapeutic intervention in other patients with this disease.

It is not clear why lysine and proline concentrations were increased but both of these amino acids are metabolized by mitochondrial dehydrogenases which might reasonably be expected to be compromised by either. NADH/NAD ratios or as the result of a more generalized mitochondrial dysfunction to which the mitochondria of such patients could be prone, on the basis of aberrant ratios of essential mitochondrial metabolites.

The liver pathology of PC deficiency is not well known. Saudubray et al. (8) described hepatomegaly with steatosis. The pathology of the present case is relatively specific in resembling both hereditary fructose intolerance and galactosemia. All these entities share similar features such as cholestasis, steatosis, septal fibrosis, ductular proliferation, and pseudoglandular transformation of hepatocytes. The histopathological overlap of these three diseases necessitates consideration as to whether they share common pathophysiological mechanisms. We believe that the common factor in all of these diseases may be a generalized ATP deficit. In the case of hereditary fructose intolerance and galactosemia, the "active" phosphate is trapped in molecules which have been phosphorylated using ATP (i.e. fructose-1-phosphate and galactose-1-phosphate, respectively). As we have discussed, in the case of CRM negative PC deficiency there is reduced efficiency of ATP generation secondary to lack of oxaloacetate for the Kreb's cycle. The abnormal ultrastructural changes of the mitochondria could also be accounted for by the ATP deficit together with the disturbed redox potential. The hepatic steatosis would suggest a block in fatty acid $\beta$ oxidation. The cholestasis in part could be explained by the effect of ATP deficiency on the pericanulicular microfilaments (12).

The neuropathology of this infant is secondary and can be assigned to three groups. The cavitated infarcts and gliosis of the white matter reflect hypoxic-ischemic injury due to perfusion failure and are not due to congential lactic acidosis as suggested by Farkas-Bargeton et al. (9). The hemorrhages in the subependymal area over the caudate nucleus are usually seen in infants less than 32 to 33 wk gestation but they can occur up to term gestation and in this case the infant's bleeding diathesis probably contributed. The initial hemorrhages and sequelae of the hydrocephalus, synechiae, and arachnoiditis were not caused by lactic acidosis. The myelin was deficient and this is probably a nonspecific effect of the metabolic derangement rather than due to the congential lactic acidosis as such.

In our discussion we have not emphasized the hypoglycemia consequent to a lack of PC activity. This has been adequately covered in previous reports but the block in gluconeogenesis must also play a role in the pathogenesis of this disease.

We believe that in this patient it is possible to unite many disparate clinical, biochemical, and histopathological findings into a coherent chain of events following from the single main enzymatic problem. It is rare to be able to join together the sequelae; in so doing we have been encouraged to publish our findings and speculate on this interesting case.

It has been suggested (3) the severity of our patient's disorder is a reflection of complete absence of PC activity in a CRM negative patient. Whether there will be equally severe disease in the occasional CRM positive patient will become apparent as more cases are investigated. It is our hope that our report will not only help in understanding the effects of PC deficiency, but will also help others in identification and diagnosis of such patients and in the development of appropriate management.

\section{REFERENCES}

1. Coude FX, Ogier H, Marsac C, Munnich A, Charpentier C, Saudubray JM 1981 Secondary citrullinemia with hyperammonemia in four neonatal cases of pyruvate carboxylase deficiency. Pediatrics 68:914

2. Charpenter C, Tetau JM, Ogier H, Saudubray JM, Coude FX, Lemonnier A 1982 Amino acid profile in pyruvate carboxylase deficiency: comparison with some other metabolic disorders. J Inherited Metab Dis 5(suppl 1):1112

3. Robinson BH, Oei J, Sherwood WG, Applegarth D, Wong LTK, Haworth J, Goodyer P, Casey R, Zaleski LA 1984 The molecular basis for the two different clinical presentations of classical pyruvate carboxylase deficiency. Am J Hum Genet 36:283-294

4. Applegarth DA, Edelsten AD, Wong LTK, Morrison BJ 1979 Observed range of assay values for plasma and cerebrospinal fluid amino acid levels in infants and children aged 3 months to 10 years. Clin Biochem 12:173-178

5. Wong LTK, Hardwick DF, Applegarth DA, Davidson AGF 1979 Reviews of metabolic screening program of Children's Hospital, Vancouver, British Columbia, 1971-1977. Clin Biochem 12:167-172

6. Applegarth DA, Wong LTK, Wong J 1980 Investigation of biochemical disease in Children's Hospital using GC/MS Hewlett-Packard GC/MS application note 176-37, May 1980. Hewlett-Packard, Palo Alto, CA

7. Persson B 1969 Determinations of plasma acetoacetate and D- $\beta$-hydroxybutyrate in new-born infants by an enzymatic fluorometric micro-method. Scand J Clin Invest 25:9-18

8. Saudubray JM, Marsac C, Charpentier C, Cathelineau L, Besson Leaud M, Leroux JP 1976 Neonatal congenital lactic acidosis with pyruvate carboxylase deficiency in two siblings. Acta Pediatr Scand 65:717-724

9. Farkas-Bargeton E, Goutieres F, Richardet JM, Thieffry S, Brissaud HE 1971 Leucoencéphalopathie familiale associete à une acidose lactique congènitale. Acta Neuropathol 17:156-168

10. Rorke LB 1983 Pathology of Perinatal Brain Injury. Raven Press, New York, pp 55-56

11. Robinson BH, Toone JR, Benedict RP, Dimmick JE, Oei J, Applegarth DA 1985 Prenatal diagnosis of pyruvate carboxylase deficiency. Prenat Diag 5:67-71

12. French SW 1976 Is cholestasis due to microfilamental failure? Hum Pathol $7: 243$ 